\title{
Performance Enhancement of Flip-Chip Packaged AlGaN/GaN HEMTs Using Active-Region Bumps-Induced Piezoelectric Effect
}

\author{
Szu-Ping Tsai, Heng-Tung Hsu, Senior Member, IEEE, Che-Yang Chiang, Yung-Yi Tu, Chia-Hua Chang, \\ Ting-En Hsieh, Huan-Chung Wang, Shih-Chien Liu, and Edward Yi Chang, Fellow, IEEE
}

\begin{abstract}
We experimentally investigated the impact of different bump patterns on the output electrical characteristics of flip-chip (FC) bonded AIGaN/GaN high-electron mobility transistors in this letter. The bump patterns were designed and intended to provide different levels of tensile stress due to the mismatch in the coefficient of thermal expansion between the materials. After FC packaging, a maximum increase of $4.3 \%$ in saturation current was achieved compared with the bare die when proper arrangement of the bumps in active region was designed. In other words, a $17 \%$ improvement has been observed on the optimized bump pattern over the conventional bump pattern. To the best of our knowledge, this is the first letter that investigates the piezoelectric effect induced by FC bumps leading to the enhancement in device characteristics after packaging.
\end{abstract}

Index Terms-AlGaN/GaN, coefficient of thermal expansion (CTE), flip-chip (FC), high-electron mobility transistors (HEMTs), tensile strain.

\section{INTRODUCTION}

C OMPARED to other III-V devices, AlGaN/GaN high-electron mobility transistors (HEMTs) have several advantages such as high breakdown field and high current density. Due to their superior properties, AlGaN/GaN HEMTs have demonstrated great potential for realizing high power switching devices for automotives, base station of cellular systems and phased array radar [1], [2].

Many researchers have studied the piezoelectric effects of $\mathrm{AlGaN} / \mathrm{GaN}$ HEMTs to investigate the variation of electrical characteristics due to external stress/strain. The channel conductance of $\mathrm{AlGaN} / \mathrm{GaN}$ HEMTs had been proved

Manuscript received April 14, 2014; revised April 29, 2014; accepted May 9, 2014. Date of publication May 29, 2014; date of current version June 24, 2014. This work was supported in part by the Ministry of Science and Technology, NCTU-UCB I-RiCE Program, and in part by the National Science Council of Taiwan, under Grant MOST-103-2911-I-009-302 and Grant 102-2221-E-155-081. The review of this letter was arranged by Editor R. Quay.

S.-P. Tsai, Y.-Y. Tu, C.-H. Chang, T.-E. Hsieh, H.-C. Wang, and S.-C. Liu are with the Department of Materials Science and Engineering, National Chiao Tung University, Hsinchu 30010, Taiwan (e-mail: mai.mse96g@g2.nctu.edu.tw).

H.-T. Hsu and C.-Y. Chiang are with the Department of Communications Engineering, Yuan Ze University, Taoyuan 320, Taiwan.

E. Y. Chang is with the Department of Materials Science and Engineering and the Department of Electronics Engineering, National Chiao Tung University, Hsinchu 30010, Taiwan.

Color versions of one or more of the figures in this letter are available online at http://ieeexplore.iee.org.

Digital Object Identifier 10.1109/LED.2014.2324619

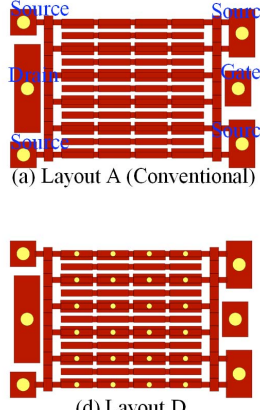

(d) Lavout D

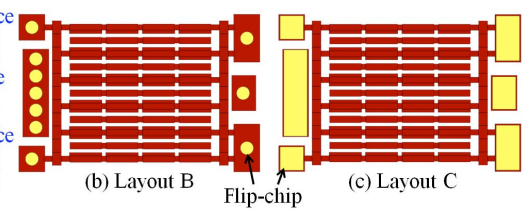

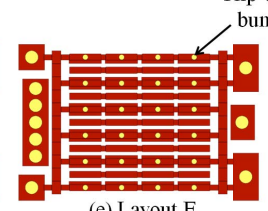

(e) Layout $\mathrm{E}$

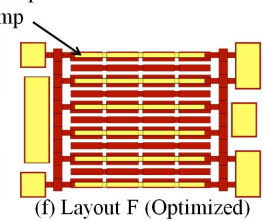

Fig. 1. Layouts of the six different bump patterns on AlN substrate.

increased during the application of tensile stress, whereas decreased during the application of compressive stress [3]. In reference [4], the biaxial tensile stress induced by thick $\mathrm{Si}_{3} \mathrm{~N}_{4}$ passivation contributed to the increase of sheet charge density leading to an increase of $11.5 \%$ in the maximum drain current compared to unpassivated devices. Unilateral tensile strain was applied to unpassivated AlGaN/GaN HEMTs to achieve $3.4 \%$ increase in saturation current in [5]. The mechanical stress was exerted on the devices through external fixture. Despite the promising feature of the increase in saturation current, integrating the fixture with devices might not be practical for real implementation.

Optimized flip-chip (FC) design with solder bumps placed directly onto both source and drain ohmic contacts was proposed in [6] to improve the thermal performance of AlGaN/GaN HEMTs bonded onto AlN substrate. Experimental results exhibited that the devices with active-region bumps can be operated at 2.6 times higher power than the conventional design to reach the same peak temperature. While the application of active-region bumps was focusing on the improvement of the thermal properties of the packaged devices, none of the previous publications has ever contemplated the effect of the tensile stress induced in the devices.

In this letter, we study the piezoelectric effect induced by the tensile stress from active-region bumps on the electrical properties of the FC packaged HEMT. Several bump patterns were designed to provide different stress states and the resultant effects on the device characteristics were compared. In order to rigorously examine the effect of the induced stress only, we applied pulsed current-voltage $(I-V)$ measurement to avoid the self-heating effect of devices and the 

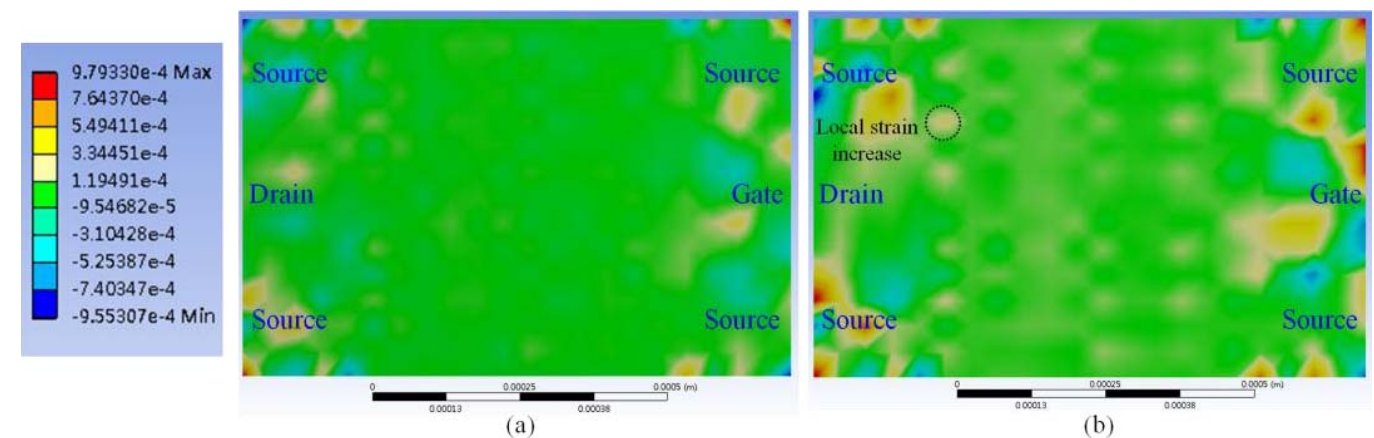

Fig. 2. Simulated strain distribution map of FC bonded AlGaN/GaN HEMTs with (a) conventional bump pattern and (b) optimized bump pattern.

quasi-isothermal condition was achieved by choosing a sufficiently short duty cycle as suggested in [7] and [8].

\section{EXPERIMENTS}

The AlGaN/GaN HEMT heterostructure was grown on a $1000-\mu$ m-thick silicon substrate using metal-organic chemical vapor deposition (MOCVD). The epitaxial layers consisted of, from bottom to top, a 120-nm-thick AlN buffer, a $5.5-\mu \mathrm{m}$-thick GaN channel layer, a 25 -nm-thick AlGaN barrier layer, and finally a 4-nm-thick GaN cap layer. The 10 -finger transistors with $2-\mu \mathrm{m}$ gate length and $500-\mu \mathrm{m}$ gate width were then fabricated using a conventional HEMT process.

Fig. 1 shows the layouts of the six bump patterns designed and fabricated on AlN ceramic substrates. They are overlaid with the device pattern for easier visualization. The patterns in Fig. 1(a), (b), and (c) contain gold electroplated pillar bumps placed on the source, gate and drain contact pads only without any active-region bumps. The radius and height were both $25 \mu \mathrm{m}$ for the cylindrical bumps in Fig. 1(a), and (b) and the only difference is the number of bumps on the drain contact pads. Rectangular bumps were used in Fig. 1(c) instead of cylindrical bumps that covered the complete source, gate and drain contact pads. As for Fig. 1(d) and (e), twenty-four pillar bumps with $10-\mu \mathrm{m}$ radius were placed directly on the active source ohmic region in addition to the original bumps on the contact pads. Ultimately, for the optimized bump pattern as shown in Fig. 1(f), six rectangular bumps with $18-\mu$ m width and $500-\mu \mathrm{m}$ length were added to the source fingers in the active region. These bump patterns were designed to induce increasing levels of tensile stress, with the optimized case gives the highest strain, as verified through numerical simulations.

\section{RESUlTS AND DISCUSSION}

Fig. 2 shows the simulated strain maps of $A 1 G a N / G a N$ HEMTs FC bonded onto the conventional and the optimized bump patterns. The strain distributions are non-homogeneous because the bumps are not uniformly distributed. From the color legend in the simulation results, the strains in the active region show no obvious variations for the conventional case, but some local increases (dashed circle in Fig. 2(b) as one example) for the optimized case. This proves that bump layout have a significant effect on the resulting strain distribution.
The DC and pulsed $I_{D}-V_{D}$ measurements were performed by Agilent B1505A power device analyzer. Figs. 3(a) and (b) show the DC $I_{D}-V_{D}$ characteristics of the $5 \mathrm{~mm} \mathrm{AlGaN/GaN}$ HEMT FC bonded with the conventional and the optimized bump patterns. We define $I_{D, \max }$ as the saturation drain current at $V_{G}=1 \mathrm{~V}$. From the DC measurement results, the $I_{D, \max }$ of the $5 \mathrm{~mm} \mathrm{AlGaN} / \mathrm{GaN}$ HEMT bare die was $850 \mathrm{~mA}$. The self-heating effect can be observed from the negative slopes in $I_{D}-V_{D}$ curves under high bias conditions. After packaging, the maximum drain current could only be measured to $1 \mathrm{~A}$ (Fig. 3(b)) due to the current limit of Agilent B1505A in DC mode. The observed performance changes after packaging can be characterized by $\Delta I_{D, \max }(\%)=\left[I_{D, \max }\right.$ (package) $I_{D, \max }$ (bare die)] $/ I_{D, \max }$ (bare die). As can be seen in Fig. 3(c), all the maximum drain currents of the six bump patterns were improved after FC packaging. That is because the thermal effect dominates in DC measurement and the FC bumps can help to dissipate heat. To investigate the stress effect only, we use pulsed $I_{D}-V_{D}$ measurement with a sufficiently short duty cycle, which is $1 \%$, to eliminate the thermal effect.

Figs. 4 (a) and (b) show the pulsed $I_{D}-V_{D}$ measurement results of the FC bonded AlGaN/GaN HEMTs with the conventional and the optimized bump layouts, respectively. Fig. 4(c) shows the $\Delta I_{D, \max }(\%)$ of the six different FC packages. They are $-12.7 \%,-4.3 \%$, and $-1.2 \%$ respectively for the cases corresponding to bump patterns shown in Fig. 1(a), (b) and (c) where the bumps were connected to the contact pads only. On the contrary, instead of performance degradation, we observed increases in the maximum drain current of $1.1 \%$, $1.2 \%$, and $4.3 \%$ for the cases with bump patterns shown in Fig. 1(d), (e), and (f). A positive $\Delta I_{D, \max }(\%)$ represents an improvement of device performance after packaging. In these cases, we have bumps connected to the source ohmic region in addition to those placed on contact pads.

From the transfer characteristics of the AlGaN/GaN HEMT and its FC package, a small threshold voltage $\left(V_{t h}\right)$ shift (within 3\%) toward more negative value has been observed after FC bonding (data not shown). Such $V_{t h}$ change as well as the enhancement in the maximum drain current should be attributed to the induced piezoelectric effect which leads to the increase in the sheet charges similar to the mechanism discussed in [5].

Since the coefficient of thermal expansion (CTE) of $\mathrm{Au}$ $\left(14.2 \mathrm{ppm} /{ }^{\circ} \mathrm{C}\right.$ is larger than that of $\mathrm{GaN}\left(5.6 \mathrm{ppm} /{ }^{\circ} \mathrm{C}\right.$, a tensile 


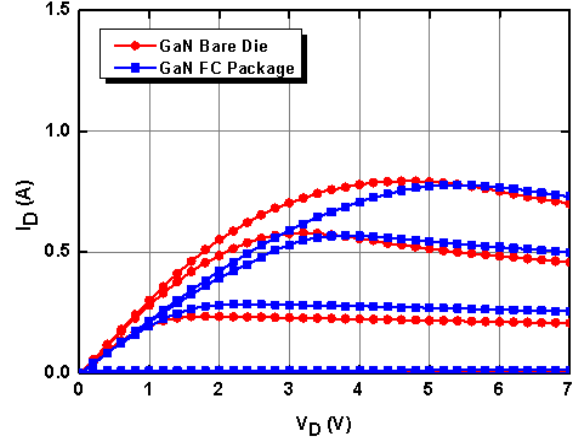

(a)

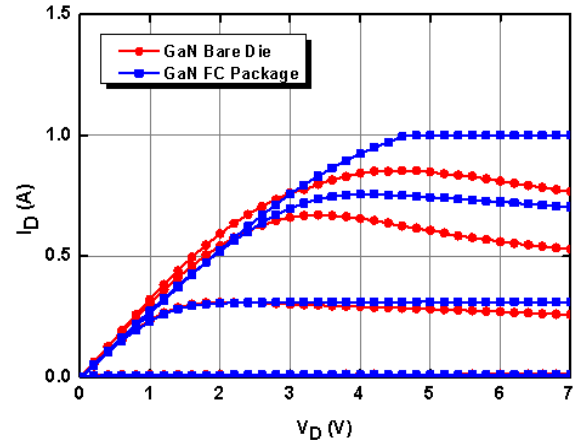

(b)

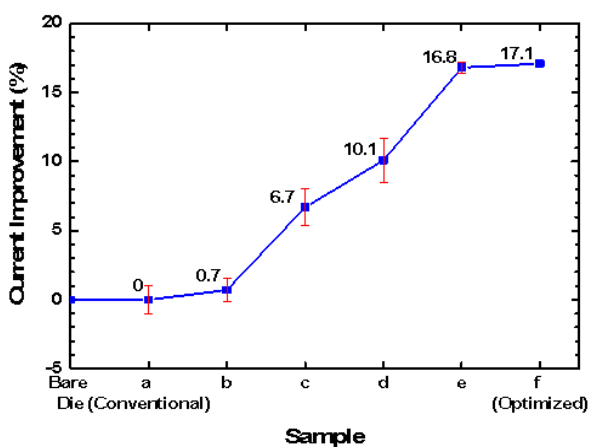

(c)

Fig. 3. DC $I_{D}-V_{D}$ measurements of FC bonded AlGaN/GaN HEMTs with (a) conventional bump pattern and (b) optimized bump pattern. (c) Improvements on $I_{D, \max }$ of the six different FC packages. (Note that no error bar for the optimized case since the $I_{d, \max }$ were all $1 \mathrm{~A}$ after FC packaging due to the current limit of Agilent B1505A in DC mode.)

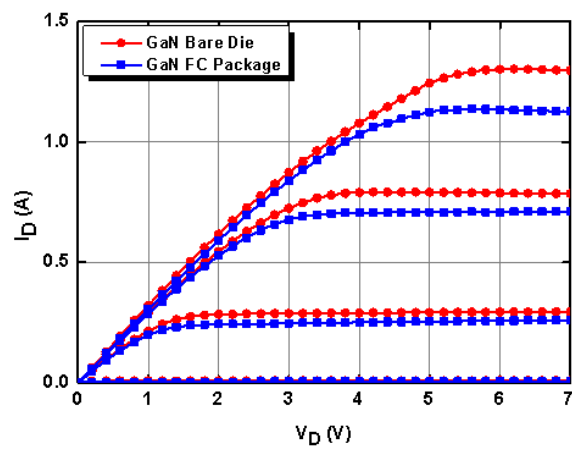

(a)

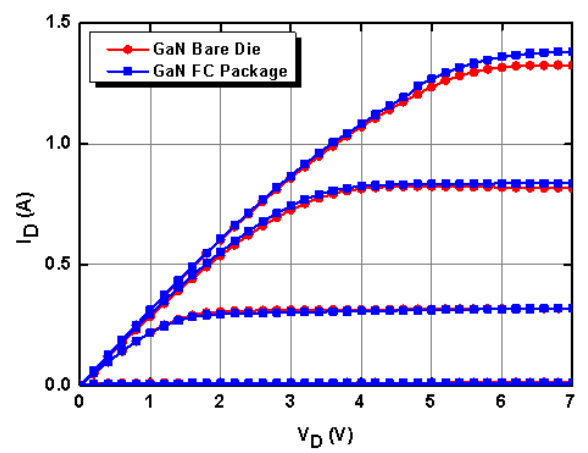

(b)

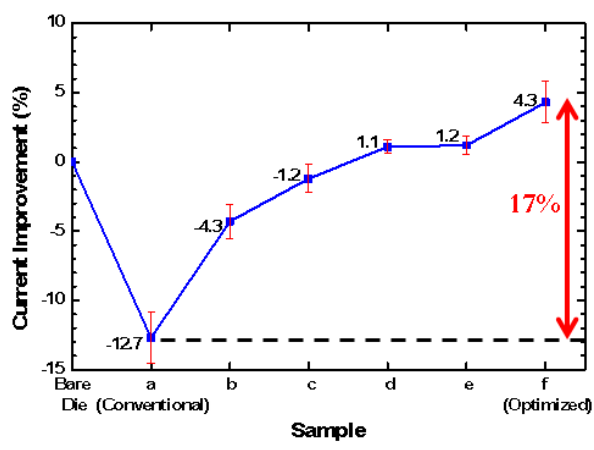

(c)

Fig. 4. Pulsed $I_{D}-V_{D}$ measurements of FC bonded AlGaN/GaN HEMTs with (a) conventional bump pattern and (b) optimized bump pattern. (c) Improvements on $I_{D, \max }$ of the six different FC packages.

stress will be induced in the active area of the transistor after FC bonding for the cases with active-region bumps. Therefore, the main reason for the increase in the drain current was due to the additional piezoelectric charges produced in the HEMT channel during device operation. Furthermore, the simulated mechanical strain for the optimized bump patterns exhibits the same order of magnitude as the experimental data in [5] resulting in a comparable increase in the maximum drain current. We thus believe that the optimized bump pattern design can effectively replace the external bending fixture adopted in [5]. It should also be noted that adding more bumps under the active region certainly contributes to the improved heat dissipation. Therefore, the active-region bumps act not only as heat sinks but also act as the bending fixture as reported in [5] that could modulate the channel current density.

\section{CONCLUSION}

We have successfully demonstrated the improvement in the electrical characteristics of the FC bonded $\mathrm{AlGaN} / \mathrm{GaN}$ HEMTs through the optimization of bump pattern design in the active region. As compared to the conventional FC bump pattern, a total improvement of $17 \%$ in maxima drain current has been achieved from the optimized design. Such improvement is mainly attributed to the tensile stress induced by the bumps in the active-region. The results showing that the device electrical performance of FC packaged $\mathrm{AlGaN} / \mathrm{GaN}$ HEMT can be improved by proper bump design to take advantage of the piezoelectric effect.

\section{ACKNOWLEDGMENT}

The authors would like to thank B. Y. Chen and G. W. Huang at the High Frequency Technology Center, National Nano Device Laboratories, Hsinchu, Taiwan, for their support on pulsed I-V measurements.

\section{REFERENCES}

[1] A. Chini et al., "12 W/mm power density AlGaN/GaN HEMTs on sapphire substrate," Electron. Lett., vol. 40, no. 1, pp. 73-74, Jan. 2004.

[2] Y.-F. Wu et al., "30-W/mm GaN HEMTs by field plate optimization," IEEE Electron Device Lett., vol. 25, no. 3, pp. 117-119, Mar. 2004.

[3] B. S. Kang et al., "Effect of external strain on the conductivity of $\mathrm{AlGaN} / \mathrm{GaN}$ high-electron-mobility transistors," Appl. Phys. Lett., vol. 83, no. 23, pp. 4845-4847, Dec. 2003.

[4] C. M. Jeon and J.-L. Lee, "Effects of tensile stress induced by silicon nitride passivation on electrical characteristics of $\mathrm{AlGaN} / \mathrm{GaN}$ heterostructure field-effect transistors," Appl. Phys. Lett., vol. 86, no. 17, pp. 172101-1-172101-3, Apr. 2005.

[5] C.-T. Chang et al., "Changes of electrical characteristics for AlGaN/GaN HEMTs under uniaxial tensile strain," IEEE Electron Device Lett., vol. 30, no. 3, pp. 213-215, Mar. 2009.

[6] J. Das et al., "Improved thermal performance of AlGaN/GaN HEMTs by an optimized flip-chip design," IEEE Trans. Electron Devices, vol. 53, no. 11, pp. 2696-2702, Nov. 2006.

[7] J. Scott et al., "Pulsed device measurements and applications," IEEE Trans. Microw. Theory Techn., vol. 44, no. 12, pp. 2718-2723, Dec. 1996.

[8] A. F. A. Rahim, A. V. Kordeschl, and Y. M. Yusofe, "Self heating characterization of $32 \mathrm{~V}$ MOSFETs using pulsed gate measurement," in Proc. IEEE ICSE, Kuala Lumpur, Malaysia, Dec. 2006, pp. 361-366. 